Article

\title{
Visible-Light-Excited Room Temperature Phosphorescent Carbon Dots
}

\author{
Sizhe Hu ${ }^{1,2}$, Kai Jiang ${ }^{2, *}$, Yuci Wang ${ }^{2,3}$, Sui Wang ${ }^{1}$, Zhongjun $\mathrm{Li}^{3}$ and Hengwei Lin ${ }^{2,4, *(1)}$ \\ 1 School of Materials Science and Chemical Engineering, Ningbo University, Ningbo 315211, China; \\ husizhe@nimte.ac.cn (S.H.); wangsui@nbu.edu.cn (S.W.) \\ 2 Ningbo Institute of Materials Technology \& Engineering (NIMTE), Chinese Academy of Sciences, \\ Ningbo 315201, China; wangyuci@nimte.ac.cn \\ 3 College of Chemistry and Molecular Engineering, Zhengzhou University, Zhengzhou 450001, China; \\ lizhongjun@zzu.edu.cn \\ 4 International Joint Research Center for Photo-responsive Molecules and Materials, School of Chemical and \\ Material Engineering, Jiangnan University, Wuxi 214122, China \\ * $\quad$ Correspondence: jiangkai@nimte.ac.cn (K.J.); linhengwei@nimte.ac.cn (H.L.); Tel.: +86-574-8668-5130 (K.J.)
}

Received: 6 February 2020; Accepted: 29 February 2020; Published: 4 March 2020

\begin{abstract}
Carbon dots (CDs) with a room temperature phosphorescent (RTP) feature have attracted considerable interest in recent years due to their fundamental importance and promising applications. However, the reported matrix-free RTP CDs only show short-wavelength (green to yellow) emissions and have to be triggered by ultraviolet (UV) light (below $400 \mathrm{~nm}$ ), limiting their applications in certain fields. Herein, visible-light-excited matrix-free RTP CDs (named AA-CDs) with a long-wavelength (orange) emission are reported for the first time. The AA-CDs can be facilely prepared via a microwave heating treatment of L-aspartic acid (AA) in the presence of ammonia and they emit unique orange RTP in the solid state with visible light $(420 \mathrm{~nm})$ excitation just being switched off. Through the studies of the carbonization process, the $\mathrm{C}=\mathrm{O}$ and $\mathrm{C}=\mathrm{N}$ containing moieties in the AA-CDs are confirmed to be responsible for the observed RTP emission. Finally, the applications of AA-CDs in information encryption and anti-counterfeiting were preliminarily demonstrated.
\end{abstract}

Keywords: carbon dots; room temperature phosphorescence; visible-light-excitation; anti-counterfeiting

\section{Introduction}

Room temperature phosphorescent (RTP) materials have been continuously stimulating extensive research interests in recent years due to their significance in many fields of applications, such as optoelectronic devices, chemical sensing, bioimaging, anticounterfeiting, etc. [1-8]. The traditional pure-organic-small-molecule-based RTP materials, however, usually exhibit the shortcomings of complicated synthesis and purification, and require a highly crystallized form of compounds to produce RTP [2,4,9-11]. Moreover, most of the reported pure organic RTP materials have to be excited by ultraviolet (UV) light (below $400 \mathrm{~nm}$ ), which significantly limits their range of applications (e.g., being not suitable for biology-relevant fields) [12]. In comparison with UV light, visible light shows less phototoxicity and deeper penetrability, which is more preferred for triggering RTP [13-15]. Therefore, it is still highly desirable to design and synthesize new classes of RTP materials with facile preparation process and visible-light excitable properties.

As a new type of luminescent nanomaterial, carbon dots (CDs) have received extensive attention due to numerous superior properties, such as easy preparation, low toxicity, high photostability, tunable emission, etc. [13,16-24]. Interestingly, RTP phenomena of CDs have been discovered recently by embedding or immobilizing in a variety of matrices, including poly (vinyl alcohol), polyurethane, 
layered double hydroxides, urea/biuret, zeolites and so on [25-36]. Although the introduction of matrices could realize RTP of CDs, the inherent chemical and physical properties of matrices would hinder applications of the RTP properties of CDs. More importantly, matrix-free RTP CDs have also been prepared very recently based on the concept of crosslink enhanced emission (CEE), self-immobilization fluorophores and specific elements (e.g., N, P and halogens) doping [37-47]. The reported matrix-free RTP CDs, however, only showed RTP emission colors from green to yellow and have to be excited by UV light as well [37-47]. To further expand the applications, it is significant to develop matrix-free RTP CDs holding long-wavelength (orange to red, and even to near infrared (NIR)) emissions and capable of being excited by visible-light, but this is still a formidable challenge.

Based on the prerequisites for activating RTP in organic compounds and matrix-free CDs (e.g., introducing heteroatom to improve intersystem crossing (ISC) and producing matrix-like structures to self-immobilize fluorophores in CDs), [38-41] herein, a facile and quick method is reported to prepare visible-light-excited matrix-free RTP CDs (named AA-CDs) with orange emission. As shown in Figure 1a, L-aspartic acid (AA) was taken as the carbon precursor, and the AA-CDs can be obtained by microwave-irradiation heating AA in the presence of ammonia water (see Materials and Methods section for details). Notably, other kinds of natural amino acids do not produce RTP CDs by the same reaction conditions; this is because that AA could form crosslinked polymer-like structures under high temperatures and thus self-immobilize fluorophores in the AA-CDs [48,49]. A dilute aqueous dispersion of the purified AA-CDs exbibits common blue fluorescence (FL), like many other reported CDs, under the irradiation of a UV lamp (365 nm) (Figure 1b). Impressively, the solid powder of AA-CDs displays a warm white emission under a commercial blue-light LED (420 nm), and an orange afterglow is observed and lasts for several seconds to naked eye with the LED being just switched off (Figure 1c). To the best of our knowledge, this is the first example of achieving visible-light-excited matrix-free CDs with a long-wavelength (orange) afterglow.

a)

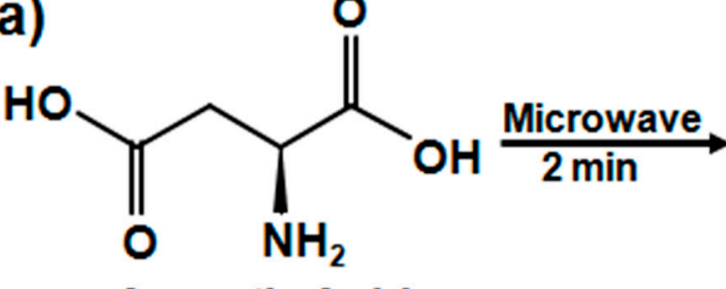

c)
Aspartic Acid b)

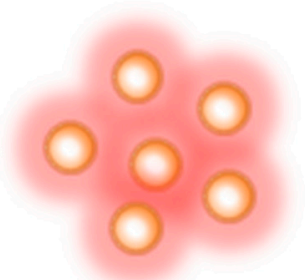

AA-CDs
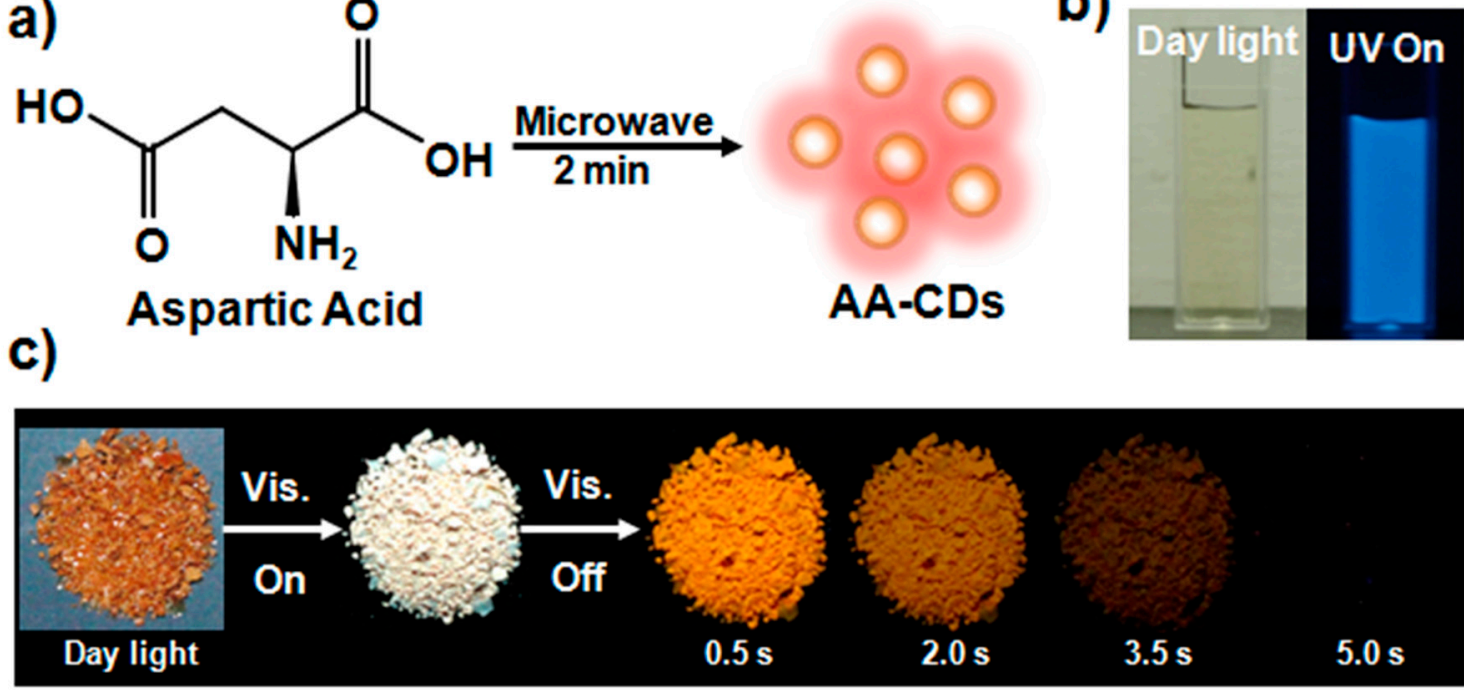

Figure 1. (a) Schematic illustration of the preparation process for visible-light-excited matrix-free room temperature phosphorescent (RTP)carbon dots (CDs) named AA-CDs. (b) Photographs of AA-CDs water dispersion under daylight and ultraviolet(UV) light $(365 \mathrm{~nm})$. (c) Photographs of the AA-CDs powder under the daylight, a visible light LED $(420 \mathrm{~nm})$ and delay time after ceasing the LED irradiation. 


\section{Materials and Methods}

\subsection{Reagents}

Reagent grade of L-aspartic acid (AA) was purchased from Aladdin Chemicals Co. Ltd. (Shanghai, China). An ammonia solution of superior purity (25-28\%) was purchased from Sinopharm Chemical Reagent Co. Ltd. (Shanghai, China). All chemicals were used as received without further purification unless otherwise specified. Deionized (DI) water was used throughout this study.

\subsection{Synthesis of $A A-C D$ s and $A A-C D s-2$}

Typically, $2.0 \mathrm{~mL}$ of ammonia solution was dissolved in $8 \mathrm{~mL}$ of DI water, and then $1500 \mathrm{mg}$ of AA was slowly added into this solution with stirring, and the precursor was completely dissolved by ultrasonic treatment for $15 \mathrm{~min}$. The as-formed homogeneous and transparent solution was transferred into a beaker and heated in a domestic microwave oven for about $2 \mathrm{~min}(750 \mathrm{~W})$. After cooling to room temperature, the crude burnt yellow gel-like solid was obtained and could be completely dissolved by the addition of a sodium carbonate solution. For purifying the CDs, the above aqueous solution was firstly centrifuged $(10,000 \mathrm{rpm} / \mathrm{min}$ for $20 \mathrm{~min})$ and filtered through a $0.22 \mu \mathrm{m}$ membrane filter to remove large or agglomerated particles, then, the supernatant was collected and subjected to dialysis (MWCO: $1000 \mathrm{Da}$ ) for 3 days. Finally, the purified AA-CDs powder could be obtained by freeze-drying. Please note that the concentrations of AA and ammonia are critical for the successful preparation of AA-CDs. For example, solid-state white emissive CDs could be prepared by a similar procedure if lower concentrations of AA and ammonia were being used [48].

In order to form the further carbonized samples (AA-CDs-2), the crude, burnt, yellow gel-like solid was re-dissolved by the addition of DI water $(8 \mathrm{~mL})$ and ammonia solution $(2 \mathrm{~mL})$ and by then continuing to heat it in a domestic microwave oven for about $2 \mathrm{~min}(750 \mathrm{~W})$. The above treatments were repeated three more times until a dark-brown gel-like solid was formed. The purifying procedure for the AA-CDs-2 was as the same as that of the AA-CDs, and the solid AA-CDs-2 powder could then be obtained by freeze-drying it.

\subsection{Procedure}

Preparation of the security ink: Typically, the AA-CDs aqueous dispersion $(150 \mathrm{mg} / \mathrm{mL})$ was used directly as security ink for anti-counterfeiting and information encryption.

Preparation of the interference fluorescent security ink (W-CDs): CDs with blue FL emission were prepared by a microwave assisted heating method. Typically, $0.5 \mathrm{~mL}$ of ethylenediamine was added into $20 \mathrm{~mL}$ of L-aspartic acid, aqueous dispersion $(0.05 \mathrm{~g} / \mathrm{mL})$. Then, the mixture was heated in a domestic oven for $4 \mathrm{~min}(750 \mathrm{~W})$. The excess precursors and resulting small molecules were removed by dialyzing against water through a dialysis membrane (MWCO: $1000 \mathrm{Da}$ ) for 3 days. The obtained CDs were dispersed in water $(50 \mathrm{mg} / \mathrm{mL})$ and employed as interference ink for information encryption.

\subsection{Equipment and Characterization}

Transmission electron microscopy (TEM) observations were performed on a Tecnai F20 microscope (FEI, Hillsboro, OR, USA). X-ray photoelectron spectroscopy (XPS) was carried out with ESCALAB 250Xi (Thermo Scientific, Waltham, MA, USA). Fourier transform infrared (FT-IR) spectra were obtained on a Nicolet 6700 FT-IR spectrometer (Thermo Nicolet Corp., Madison, WI, USA). Photoluminescence, phosphorescence emission and excitation spectra were measured on a Hitachi F-4600 spectrophotometer (Hitachi, Tokyo, Japan) at ambient conditions. UV-Vis absorption spectra were recorded on a PERSEE T10CS UV-Vis spectrophotometer (Persee, Beijing, China). FL and Phos lifetimes were measured using an EDINBURGH FLS 980photoluminescence spectrometer (Edinburgh Instruments, Wales, UK). Photographs of FL and Phos images were taken using a Canon camera (EOS 550, Tokyo, Japan) under a hand-held blue LED lamp (420 nm). PL QY measurements were carried out with a QE-2100 quantum 
efficiency measurement system (Otsuka Electronics, Tokyo, Japan).All the FL and RTP spectra and lifetimes were measured under aerobic atmosphere in this study unless otherwise noted.

\section{Results and Discussion}

\subsection{Optical Properties of Carbon Dots (CDs)}

First of all, photophysical properties of the AA-CDs are investigated in detail. The aqueous dispersion of the AA-CDs exhibits an excitation-dependent FL feature with emission maxima at $456 \mathrm{~nm}$ under the optimal excitation wavelength of $358 \mathrm{~nm}$ (Figure 2a and Figure S1 in Supplementary Materials (SM)). The absolute FL quantum yield (QY) is measured to be $22.45 \%$ using an integrated sphere (Table S1 in SM). As shown in the UV-Vis absorption spectrum (Figure 2a), AA-CDs show a distinct absorption peak at $280 \mathrm{~nm}$ and a weak shoulder peak at about $340 \mathrm{~nm}$, which can be attributed to the $\pi \rightarrow \pi^{*}$ transition of $\mathrm{C}=\mathrm{C}$ and $\mathrm{n} \rightarrow \pi^{*}$ transitions of $\mathrm{C}=\mathrm{O} / \mathrm{C}=\mathrm{N}$, respectively $[31,50]$. The good consistency between their FL excitation and absorption spectra indicates that the $\mathrm{C}=\mathrm{O} / \mathrm{C}=\mathrm{N}$ containing moieties in the AA-CDs should be responsible for the FL emission (Figure 2a). Moreover, it is found that the time-resolved FL spectrum of AA-CDs has to be fitted by a double-exponential function with an average lifetime of 9.99 ns (Figure S2 and Table S2 in SM). According to previous reports, the excitation-dependent FL emission and double-exponential decay features imply the existence of multiple emission centers within the AA-CDs [31,51-53]. In addition, the AA-CDs powder also exhibits an excitation-dependence FL feature (Figure S3 in SM) and the time-resolved spectrum has to be fitted by a double-exponential function as well. Both of the properties are the same as that of their aqueous dispersion, but with a shorter average lifetime of 8.04 ns (Figure S4 and Table S2 in SM). Impressively, an obvious orange RTP can be observed from the AA-CDs powder with visible light $(420 \mathrm{~nm})$ irradiation being just switched off (Figure 1c). Based on the RTP emission and excitation spectra (Figure $2 b$ ), the maximum RTP emission is located at $585 \mathrm{~nm}$ under the excitation wavelength of $312 \mathrm{~nm}$, and meanwhile, the RTP emissions shift from 585 to $650 \mathrm{~nm}$ with the excitation wavelengths changing from 300 to $450 \mathrm{~nm}$, indicating an excitation-dependence RTP property. Moreover, from Figure S5 (SM), it is clear to see that both FL and RTP exhibit two excitation bands at similar positions, although with different ratios, indicating that the RTP of the AA-CDs mainly arises from the same moieties as that FL. It is worthy to note here that the RTP of the AA-CDs is able to be excited by visible light, and this is very rare in pure organic RTP materials and CDs relevant systems [12]. Based on the RTP spectrum, the CIE 1931 chromaticity coordinates of AA-CDs RTP emission is determined to be $(0.51,0.45)$ under the visible light $(420 \mathrm{~nm})$ irradiation (Figure $2 \mathrm{c})$, which is located at the orange area and is in good consistence with the emission color (Figure 1c). To the best of our knowledge, this is the first example of visible-light excited matrix-free RTP CDs with a long wavelength (orange) emission. 
a)

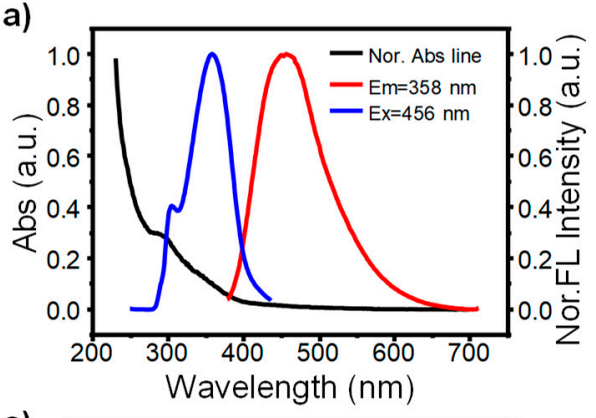

c)

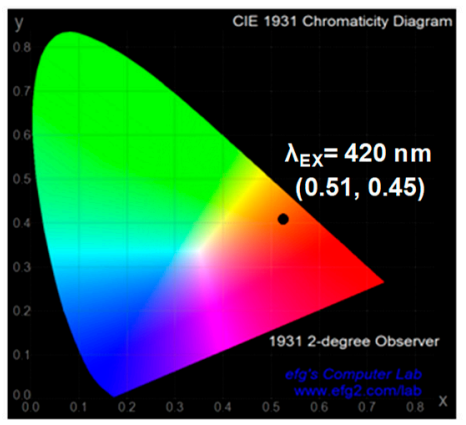

b)

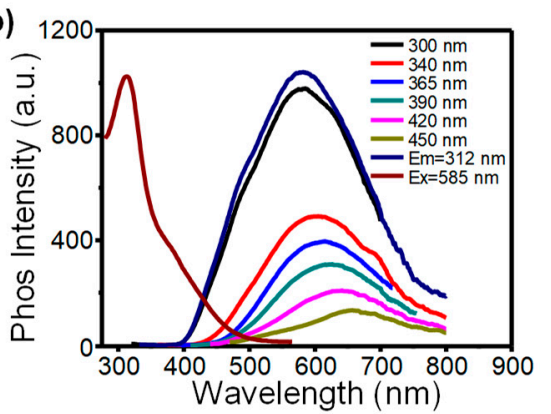

d)

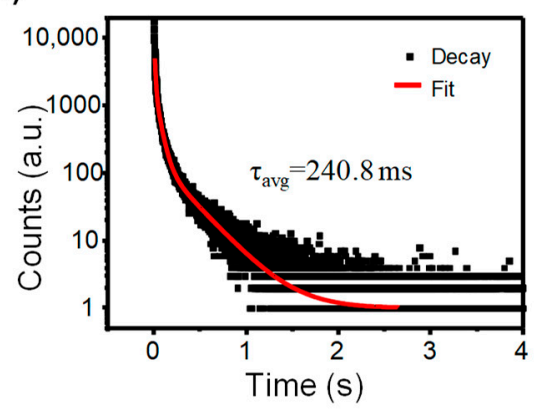

Figure 2. (a) The UV-Vis absorbance (black line), fluorescence (FL) emission (red line), and FL excitation (blue line) spectra of the AA-CDs water dispersion $(1 \mathrm{mg} / \mathrm{mL})$. (b) RTP emission spectra at different excitation wavelengths and excitation spectrum at the emission of $585 \mathrm{~nm}$ of the AA-CDs powder at ambient conditions. (c) CIE chromaticity coordinates of the AA-CDs powder under the excitation at $420 \mathrm{~nm}$. (d) RTP decay spectrum and fitting curve (red line) of the AA-CDs powder at ambient conditions.

To obtain a better understanding of the RTP properties, the RTP decay behaviors of AA-CDs powder were further examined. As shown in Figure 2d and Table S3 (SM), the RTP decay spectrum of AA-CDs powder has to be fitted with a tri-exponential function, indicating a multi-channel emissive nature. The three decay lifetime components were found to be $9.9,51.78$ and $298.76 \mathrm{~ms}$ (under excitation at $312 \mathrm{~nm}$ ), respectively (Table S3 in SM), and an average lifetime was calculated to be $240.8 \mathrm{~ms}$ on the basis of the following equation $[31,33,54]$.

$$
\tau_{a v g}=\sum \alpha_{i} \tau_{i}^{2} / \sum \alpha_{i} \tau_{i}
$$

In addition, an afterglow emission from AA-CDs aqueous dispersion is observed at a low temperature $(77 \mathrm{~K})$ (Figure S6 in SM), but not at room temperature (even under nitrogen or argon inert atmospheres). This demonstrates that the RTP emission from AA-CDs powder should mainly arise from self-immobilization of the excited triplet species in their solid form [39,41,42]. Moreover, according to the low-temperature (77 K) FL and phosphorescence spectra of AA-CDs aqueous dispersion (Figure S6 in $\mathrm{SM}$ ), an energy gap between the lowest $\mathrm{T} 1$ and $\mathrm{S} 1\left(\Delta E_{S T}\right)$ can be calculated to be $0.46 \mathrm{eV}$, and this small value of band gap is favorable for an effective ISC process to populate triplet excitations $[33,55]$.

\subsection{Morphologies and Structure Analyses of $A A-C D$ s}

Subsequently, morphology and chemical structures of the AA-CDs were examined to provide deeper insights into their optical characteristics. First, transmission electron microscopy (TEM) measurements were performed to test the morphology of AA-CDs. As shown in Figure 3a,b, the TEM image displays that AA-CDs are quasi-spherical particles with an average size of $4.8 \mathrm{~nm}$. The high resolution (HR) TEM observations confirm clear lattice fringes with a spacing of $0.21 \mathrm{~nm}$ (Figure 3c), which is in accordance with the (100) facets of graphite [56-58]. The surface groups and chemical compositions of the AA-CDs are followed and identified by Fourier transform infrared (FT-IR) and X-ray 
photoelectron spectroscopy (XPS). As seen in Figure 3d (red line), the FT-IR spectrum of AA-CDs shows obvious absorption peaks at 3432, 3273, 2939, 1712, 1659, 1400 and $1168 \mathrm{~cm}^{-1}$, which are attributed to the stretching vibrations of $\mathrm{O}-\mathrm{H}, \mathrm{N}-\mathrm{H},-\mathrm{CH} 2-, \mathrm{C}=\mathrm{O}, \mathrm{C}=\mathrm{C}, \mathrm{C}-\mathrm{N}$ and $\mathrm{C}-\mathrm{O}$ bonds, respectively $[41,48,49]$. These FT-IR analyses could be further confirmed by the XPS characterizations. As shown in Figure 3e, the AA-CDs mainly contain C, N and O elements with corresponding contents to be $59.55 \%, 13.30 \%$ and $27.15 \%$, respectively. More in detail, the HR XPS spectrum of $C 1 \mathrm{~s}$ indicates the presence of $\mathrm{C}-\mathrm{C} / \mathrm{C}=\mathrm{C}(284.1 \mathrm{eV}), \mathrm{C}-\mathrm{N}(285.9 \mathrm{eV}), \mathrm{C}-\mathrm{O}(286.9 \mathrm{eV}), \mathrm{C}=\mathrm{N} / \mathrm{C}=\mathrm{O}(288.5 \mathrm{eV})$ and $\mathrm{N}-\mathrm{C}=\mathrm{O}(289.9 \mathrm{eV})$ (Figure 3f) [49,59]. The HR XPS spectrum of N 1s contains three components that can be assigned to pyrrolic $\mathrm{N}(399.0 \mathrm{eV})$, graphite $\mathrm{N}(400.6 \mathrm{eV})$ and amino $\mathrm{N}(401.2 \mathrm{eV})$ (Figure 3g) [60,61]. The HR XPS of $\mathrm{O} 1 \mathrm{~s}$ spectrum was deconvoluted into three peaks at $530.9 \mathrm{eV}, 532.6 \mathrm{eV}$ and $533.7 \mathrm{eV}$, corresponding to $-\mathrm{OH}, \mathrm{C}=\mathrm{O}$ and $\mathrm{N}-\mathrm{C}=\mathrm{O}$, respectively (Figure $3 \mathrm{~h}$ ) $[59,62]$.
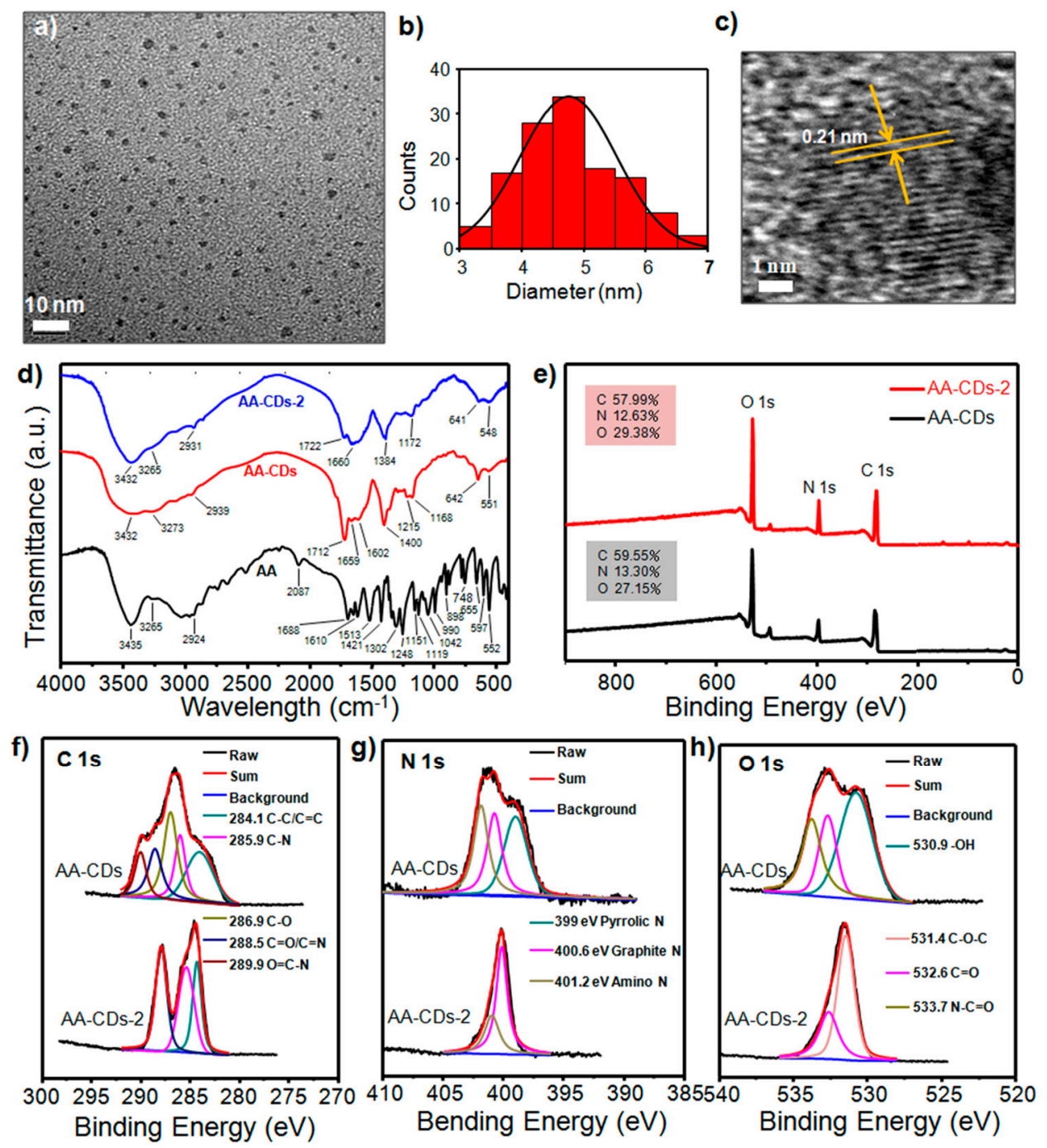

Figure 3. (a) Transmission electron microscopy (TEM) image, (b) the size histogram and (c) the high resolution TEM image of AA-CDs. (d) FT-IR spectra of L-aspartic acid (AA), AA-CDs and AA-CDs-2. (e)X-ray photoelectron spectroscopy (XPS) spectra of AA-CDs and AA-CDs-2. (f-h) High-resolution XPS and corresponding fitting results for the $\mathrm{C}$ 1s (f), $\mathrm{N}$ 1s (g), $\mathrm{O}$ 1s (h), spectra of AA-CDs and AA-CDs-2.

\subsection{Formation Process and Phosphorescence Mechanism of AA-CDs}

In order to clarify the formation process and phosphorescence origins of the AA-CDs, the carbon precursor (i.e., AA) and a further carbonized sample (named AA-CDs-2, see Materials and Methods 
section for details of synthesis) were set up as references to investigated. From the FT-IR spectra (Figure 3d), the stretching vibrations of $-\mathrm{OH}$ bond at $3435 \mathrm{~cm}^{-1}$ and carboxyl group $(\mathrm{COOH})$ at 1688 $\mathrm{cm}^{-1}$ decreased or almost disappeared, and accompanied by the emergence of carbonyl (amide $\mathrm{C}=\mathrm{O}$ ) stretching vibration at $1712 \mathrm{~cm}^{-1}$ from AA to the AA-CDs, indicating dehydration and amidation reactions occurred during the preparation process for the AA-CDs. From comparing the FT-IR spectra of AA-CDs and AA-CDs-2, one can observe the increased stretching vibration of $\mathrm{C}=\mathrm{C} / \mathrm{C}=\mathrm{N}$ at $1660 \mathrm{~cm}^{-1}$ but decreased peak intensities at 3273 and $1712 \mathrm{~cm}^{-1}$, implying deeper carbonization and deamidation happened from AA-CDs to AA-CDs-2. These speculations are further verified by the XPS analyses. As shown in Figure 3e, AA-CDs and AA-CDs-2 consist of the same element compositions, but with different ratios (Table S4, SM). Moreover, the high resolution XPS spectra (Figure 3f-h) clearly demonstrated the increases of $\mathrm{C}-\mathrm{C} / \mathrm{C}=\mathrm{C}, \mathrm{C}-\mathrm{N}$ and $\mathrm{C}=\mathrm{N} / \mathrm{C}=\mathrm{O}$, but decreases of $\mathrm{C}-\mathrm{O}, \mathrm{N}-\mathrm{C}=\mathrm{O}$ and $\mathrm{O}-\mathrm{H}$ from AA-CDs to AA-CDs-2. The corresponding fitting results in Figure $3 \mathrm{f}-\mathrm{h}$ are summarized in Table S5 (SM), which provide relatively quantitative alterations of the functional groups on AA-CDs and AA-CDs-2.

Then, the optical characteristics of AA and AA-CDs-2 were also investigated. As shown in Figure S7 (SM), the solid AA exhibits an excitation-independent FL character, while the AA-CDs-2 powder shows a similar excitation-dependence as that of the AA-CDs. Note that as the degree of carbonization increased from the AA-CDs to the AA-CDs-2, the FL excitation-dependence behavior became more obvious (Figures S1 and S8, SM). This demonstrates that with the carbonization of AA, more emissive centers are created, which should be responsible for the excitation-dependent FL character $[59,63,64]$. In addition, AA is found to emit very weak RTP in the solid state (too weak to be seen by naked eye) (Figure S9a, SM). The AA-CDs-2, however, exhibit similar RTP properties as that of the AA-CDs in the solid state (Figure S9b, SM), including the visible-light excitable feature (Figure S10, SM). The quantum yields (QYs) were found to decrease from the AA-CDs (22.45\%) to the AA-CDs-2 (18.55\%) (Table S1 in SM), implying some of the fluorophores on AA-CDs being consumed when they are subjected to further carbonization $[47,63,64]$.

According to the above discussion, formation process and RTP origins of the AA-CDs could be tentatively proposed as follows: (i) Amide condensation, crosslinking polymerization and carbonization reactions could occur during the preparation process of the AA-CDs (Figure 4a); (ii) the crosslinked structure of the AA-CDs should be responsible for their enhanced FL in solution (CEE effects) and RTP in solid state (self-immobilization of emissive moieties) in comparison with AA; (iii) the more obvious excitation-wavelength dependence emission of the AA-CDs-2 than that of the AA-CDs could be ascribed to the presence of more $\mathrm{C}=\mathrm{N}$ relevant fluorophores. The FL and RTP emission processes of the AA-CDs can be simply illustrated in Figure $4 \mathrm{~b}$. 
a)
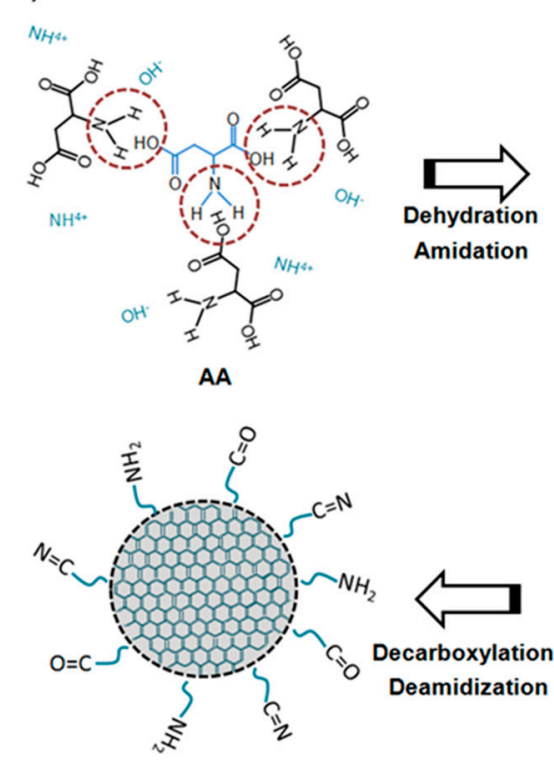

AA-CDs-2
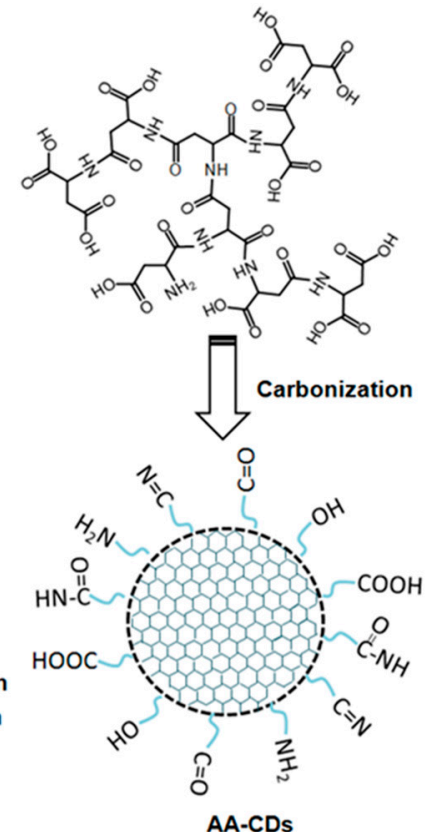

AA-CDs

b)

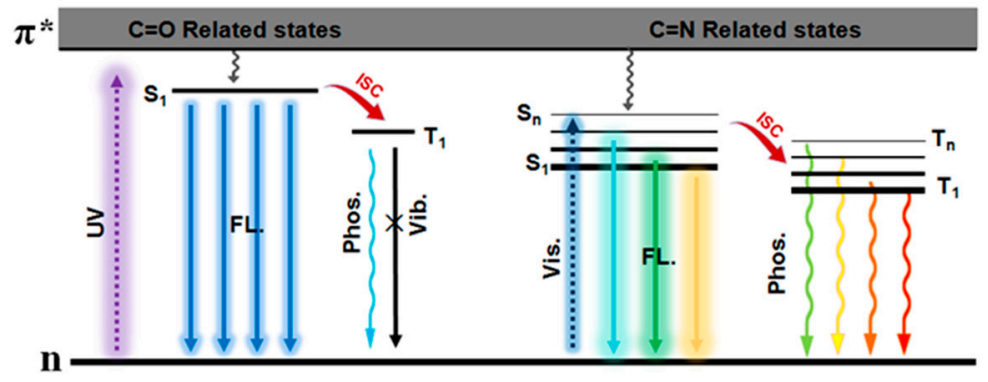

Figure 4. (a) A schematic illustration of the formation process from AA to the AA-CDs and AA-CDs-2.

(b) Proposed FL and phosphorescence emission processes of the AA-CDs.

\subsection{Applications of CDs in Anti-Counterfeiting}

Thanks to the unique visible-light-excited RTP feature, the AA-CDs are considered to be more useful in certain fields of applications. As a preliminary example, their potentials in anti-counterfeiting and information decryption is demonstrated. To present such an application, the badge of Ningbo University (made by filter paper) was firstly soaked in the aqueous dispersion of AA-CDs and then thoroughly dried. As shown in Figure 5a, the badge of Ningbo University displays white emission and yellow RTP under a visible-light LED $(420 \mathrm{~nm})$ when it is "ON" and when it has just been turned off, respectively. For the information encryption and decryption applications of the AA-CDs, another kind of white emissive FL ink (named W-CDs, see Materials and Methods section for details of synthesis) was utilized as interference. As shown in Figure 5b, wrong information (i.e., "3417") on the filter paper is observed under daylight and visible light $(420 \mathrm{~nm})$. The correct information (i.e., "1111"), however, can only be recognized under the phosphorescent mode, that is, taking the information with the visible light $(420 \mathrm{~nm})$ irradiation being just turned off. 


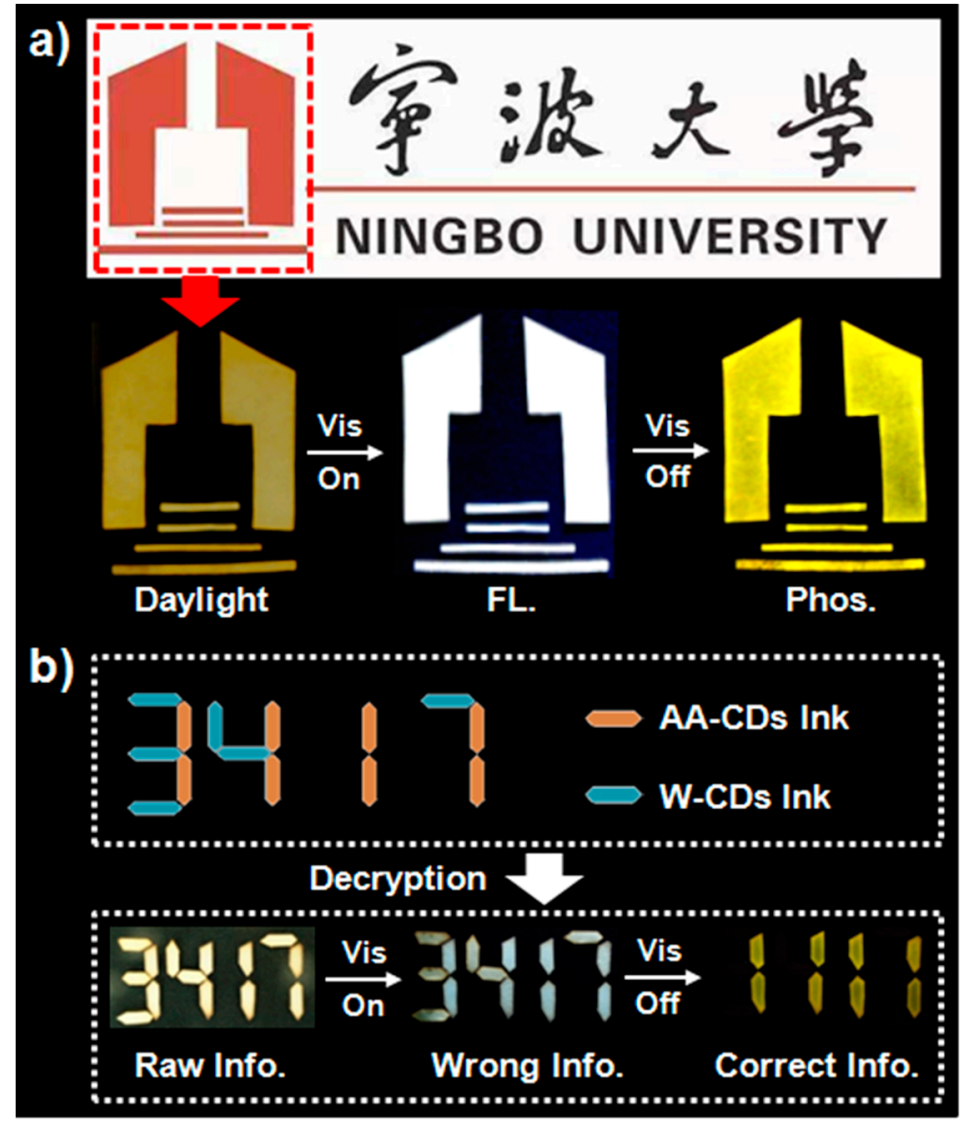

Figure 5. (a) Images of the badge of Ningbo University (made by filter paper that soaked in AA-CDs ink) under daylight, and visible light $(420 \mathrm{~nm})$ irradiation on and off, respectively. (b) Images of "3417" (made by filter paper that soaked in AA-CDs ink andinterference fluorescent security ink (W-CDs) ink) under daylight, and visible light (420 nm) irradiation on and off, respectively.

\section{Conclusions}

In summary, the preparation of visible-light-excited matrix-free RTP CDs (i.e., AA-CDs) with orange emission feature is first reported in this study. The AA-CDs aqueous dispersion exhibits common bright blue FL emission and obviously excitation-dependent properties. The powder of AA-CDs, however, displays unique warm white emission and orange RTP under visible light $(420 \mathrm{~nm})$ irradiation being on and off, respectively. Further studies revealed that the crosslinked structure of the AA-CDs should be responsible for their specific optical properties. Finally, potential applications of the AA-CDs in the fields of anti-counterfeiting and information protection were preliminarily presented. More importantly, this study expanded the excitation wavelengths from the UV region to visible-light for matrix-free RTP CDs, and this will be very beneficial for applications in certain specific fields, such as biomedicine and optoelectronic devices, and such relevant works are now ongoing in our lab.

Supplementary Materials: The following are available online at http://www.mdpi.com/2079-4991/10/3/464/s1, Figure S1: The FL emission spectra of the AA-CDs water dispersion under different excitation wavelengths and excitation spectrum at emission of $456 \mathrm{~nm}$. Figure S2: Time-resolved FL spectrum and fitting curve of AA-CDs aqueous solution $\left(\lambda_{\mathrm{ex}}=376 \mathrm{~nm}, \lambda_{\mathrm{em}}=456 \mathrm{~nm}\right)$ at ambient conditions. Figure S3: FL emission spectra of the AA-CDs powder under different excitation wavelengths and excitation spectrum at emission of $565 \mathrm{~nm}$, Figure S4: Time-resolved FL spectrum and fitting curve of AA-CDs powder $\left(\lambda_{\mathrm{ex}}=376 \mathrm{~nm}, \lambda_{\mathrm{em}}=565 \mathrm{~nm}\right)$ at ambient conditions, Figure S5: Normalized FL excitation spectrum of the AA-CDs water dispersion (1 mg/mL) and RTP excitation spectrum of AA-CDs powder in ambient conditions. Figure S6: The low temperature (77 K) FL and phosphorescence spectra of the AA-CDs water dispersion at air conditions under the excitation wavelength at 360 nm. Figure S7: (a) FL emission spectra at different excitation wavelengths and excitation spectra of AA powder, (b) FL emission spectra at different excitation wavelengths and excitation spectra of AA-CDs-2 powder. Figure S8: FL emission spectra at different excitation wavelengths and excitation spectrum at emission of wavelength $467 \mathrm{~nm}$ 
of AA-CDs-2 water dispersion. Figure S9: Phosphorescence emission spectra at different excitation wavelengths and excitation spectrum of the L-aspartic acid (a) and AA-CDs-2 (b) powders at ambient conditions. Figure S10: Photographs of the AA-CDs-2 powder at ambient conditions under day light, and visible (Vis.) light LED $\left(\lambda_{\mathrm{em}}=\right.$ $420 \mathrm{~nm}$ ) on and just being switched off, respectively. Table S1: QYs of AA-CDs and AA-CDs-2 aqueous dispersion. Table S2: Fitted parameters of the FL decay curves of the AA-CDs powder and water dispersion. Table S3: Fitted parameters of the phosphorescence decay curves of the L-aspartic acid, AA-CDs and AA-CDs-2 powder. Table S4: Relative contents of C, N and O elements of the AA-CDs and AA-CDs-2on the basis of the XPS data. Table S5: Relative contents of different functional groups in the AA-CDs and AA-CDs-2.

Author Contributions: S.H. carried out the experiments, analyzed the data and drafted the manuscript. K.J. conceived the idea, carried out the experiments, analyzed the data and drafted the manuscript. Y.W. helped in preparing the raw materials and measuring the photophysical data. S.W. and Z.L. helped in discussing and designing the experiments. H.L. perfected the idea, supervised the experiments, analyzed the data and wrote the paper. All the authors gave final approval of the version to be published and agreed to be accountable for all aspects of the work in questions related to its accuracy. All authors have read and agreed to the published version of the manuscript.

Funding: This research was funded by the National Natural Science Foundation of China (51872300 and U1832110), the China Postdoctoral Science Foundation (BX20190338), and the W. C. Wong Education Foundation (rczx0800) for financially supporting this work. The authors would also like to sincerely thank Qing Huang at NIMTE, Qiang Zhao at Nanjing University of Posts and Telecommunications (NJUPT) for allowing us accessing the fluorescence spectrophotometer and photoluminescence spectrometer in their labs, and thank Feiyang Li at NJUPT helped to measure the FL and RTP decay spectra.

Conflicts of Interest: The authors declare no conflict of interest.

\section{References}

1. Gan, N.; Shi, H.; An, Z.; Huang, W. Recent advances in polymer-based metal-free room-temperature phosphorescent materials. Adv. Funct. Mater. 2018, 28, 1802657. [CrossRef]

2. Forni, A.; Lucenti, E.; Botta, C.; Cariati, E. Metal free room temperature phosphorescence from molecular self-interactions in the solid state. J. Mater. Chem. C 2018, 6, 4603-4626. [CrossRef]

3. Zhen, X.; Tao, Y.; An, Z.; Chen, P.; Xu, C.; Chen, R.; Huang, W.; Pu, K. Ultralong phosphorescence of water-soluble organic nanoparticles for in vivo afterglow imaging. Adv. Mater. 2017, 29, 1606665. [CrossRef] [PubMed]

4. Hirata, S. Recent advances in materials with room-temperature phosphorescence: photophysics for triplet exciton stabilization. Adv. Opt. Mater. 2017, 5, 1700116. [CrossRef]

5. Zhao, W.; He, Z.; Lam, W.Y.; Peng, Q.; Ma, H.; Shuai, Z.; Bai, G.; Hao, J.; Tang, Z. Rational molecular design for achieving persistent and efficient pure organic room-temperature phosphorescence. Chem 2016, 1, 592-602. [CrossRef]

6. Xue, P.; Ding, J.; Wang, P.; Lu, R. Recent progress in the mechanochromism of phosphorescent organic molecules and metal complexes. J. Mater. Chem. C 2016, 4, 6688-6706. [CrossRef]

7. Kabe, R.; Notsuka, N.; Yoshida, K.; Adachi, C. Afterglow organic light-emitting diode. Adv. Mater. 2016, 28, 655-660. [CrossRef]

8. Mukherjee, S.; Thilagar, P. Recent advances in purely organic phosphorescent materials. Chem. Commun. 2015, 51, 10988-11003. [CrossRef]

9. Ma, X.; Xu, C.; Wang, J.; Tian, H. Amorphous pure organic polymers for heavy-atom-free efficient room-temperature phosphorescence emission. Angew. Chem. Int. Ed. 2018, 57, 10854-10858. [CrossRef]

10. An, Z.; Zheng, C.; Tao, Y.; Chen, R.; Shi, H.; Chen, T.; Wang, Z.; Li, H.; Deng, R.; Liu, X.; et al. Stabilizing triplet excited states for ultralong organic phosphorescence. Nat. Mater. 2015, 14, 685. [CrossRef]

11. Bolton, O.; Lee, K.; Kim, H.-J.; Lin, K.Y.; Kim, J. Activating efficient phosphorescence from purely organic materials by crystal design. Nat. Chem. 2011, 3, 205-210. [CrossRef] [PubMed]

12. Cai, S.; Shi, H.; Li, J.; Gu, L.; Ni, Y.; Cheng, Z.; Wang, S.; Xiong, W.-w.; Li, L.; An, Z.; et al. Visible-light-excited ultralong organic phosphorescence by manipulating intermolecular interactions. Adv. Mater. 2017, 29, 1701244. [CrossRef] [PubMed]

13. Wu, Z.; Liu, Z.; Yuan, Y. Carbon dots: Materials, Synthesis, properties and approaches to long-wavelength and multicolor emission. J. Mater. Chem. B 2017, 5, 3794-3809. [CrossRef]

14. Pan, Z.; Lu, Y.-Y.; Liu, F. Sunlight-activated long-persistent luminescence in the near-infrared from $\mathrm{Cr}^{3+}$-doped zinc gallogermanates. Nat. Mater. 2012, 11, 58-63. [CrossRef] 
15. Lian, S.; Qi, Y.; Rong, C.; Yu, L.; Zhu, A.; Yin, D.; Liu, S. Effectively leveraging solar energy through persistent dual red phosphorescence: preparation, characterization, and density functional theory study of $\mathrm{Ca}_{2} \mathrm{Zn}_{4} \mathrm{Ti}_{16} \mathrm{O}_{38}: \mathrm{Pr}^{3+}$. J. Phys. Chem. C 2010, 114, 7196-7204. [CrossRef]

16. Xiao, L.; Sun, H. Novel properties and applications of carbon nanodots. Nanoscale Horiz. 2018, 3, 565-597. [CrossRef]

17. Li, L.; Dong, T. Photoluminescence tuning in carbon dots: Surface passivation or/and functionalization, heteroatom doping. J. Mater. Chem. C 2018, 6, 7944-7970. [CrossRef]

18. Feng, T.; Zhu, S.; Zeng, Q.; Lu, S.; Tao, S.; Liu, J.; Yang, B. Supramolecular cross-link-regulated emission and related applications in polymer carbon dots. ACS Appl. Mater. Interfaces 2018, 10, 12262-12277. [CrossRef]

19. Sharma, V.; Tiwari, P.; Mobin, S.M. Sustainable carbon-dots: Recent advances in green carbon dots for sensing and bioimaging. J. Mater. Chem. B 2017, 5, 8904-8924. [CrossRef]

20. Shamsipur, M.; Barati, A.; Karami, S. Long-wavelength, multicolor, and white-light emitting carbon-based dots: Achievements made, challenges remaining, and applications. Carbon 2017, 124, 429-472. [CrossRef]

21. Yuan, F.; Li, S.; Fan, Z.; Meng, X.; Fan, L.; Yang, S. Shining carbon dots: Synthesis and biomedical and optoelectronic applications. Nano Today 2016, 11, 565-586. [CrossRef]

22. Zheng, X.T.; Ananthanarayanan, A.; Luo, K.Q.; Chen, P. Glowing graphene quantum dots and carbon dots: Properties, syntheses, and biological applications. Small 2015, 11, 1620-1636. [CrossRef] [PubMed]

23. Yang, S.-T.; Wang, X.; Wang, H.; Lu, F.; Luo, P.G.; Cao, L.; Meziani, M.J.; Liu, J.-H.; Liu, Y.; Chen, M.; et al. Carbon dots as nontoxic and high-performance fluorescence imaging agents. J. Phys. Chem. C 2009, 113, 18110-18114. [CrossRef] [PubMed]

24. Sun, Y.-P.; Zhou, B.; Lin, Y.; Wang, W.; Fernando, K.S.; Pathak, P.; Meziani, M.J.; Harruff, B.A.; Wang, X.; Wang, H. Quantum-sized carbon dots for bright and colorful photoluminescence. J. Am. Chem. Soc. 2006, 128, 7756-7757. [CrossRef] [PubMed]

25. Wang, C.; Chen, Y.; Hu, T.; Chang, Y.; Ran, G.; Wang, M.; Song, Q. Color tunable room temperature phosphorescent carbon dot based nanocomposites obtainable from multiple carbon sources via a molten salt method. Nanoscale 2019, 11, 11967-11974. [CrossRef] [PubMed]

26. Lin, C.; Zhuang, Y.; Li, W.; Zhou, T.-L.; Xie, R.-J. Blue, green, and red full-color ultralong afterglow in nitrogen-doped carbon dots. Nanoscale 2019, 11, 6584-6590. [CrossRef]

27. Li, W.; Zhou, W.; Zhou, Z.; Zhang, H.; Zhang, X.; Zhuang, J.; Liu, Y.; Lei, B.; Hu, C. A universal strategy for activating the multicolor room-temperature afterglow of carbon dots in a boric acid matrix. Angew. Chem. Int. Ed. 2019, 58, 7278-7283. [CrossRef]

28. Xia, C.; Tao, S.; Zhu, S.; Song, Y.; Feng, T.; Zeng, Q.; Liu, J.; Yang, B. Hydrothermal addition polymerization for ultrahigh-yield carbonized polymer dots with room temperature phosphorescence via nanocomposite. Chem. Eur. J. 2018, 24, 11303-11308. [CrossRef]

29. Bai, L.; Xue, N.; Zhao, Y.; Wang, X.; Lu, C.; Shi, W. Dual-mode emission of single-layered graphene quantum dots in confined nanospace: anti-counterfeiting and sensor applications. Nano Res. 2018, 11, 2034-2045. [CrossRef]

30. Liu, J.; Wang, N.; Yu, Y.; Yan, Y.; Zhang, H.; Li, J.; Yu, J. Carbon dots in zeolites: A new class of thermally activated delayed fluorescence materials with ultralong lifetimes. Sci. Adv. 2017, 3, e1603171. [CrossRef]

31. Li, Q.; Zhou, M.; Yang, Q.; Wu, Q.; Shi, J.; Gong, A.; Yang, M. Efficient room-temperature phosphorescence from nitrogen-doped carbon dots in composite matrices. Chem. Mater. 2016, 28, 8221-8227. [CrossRef]

32. Jiang, K.; Zhang, L.; Lu, J.; Xu, C.; Cai, C.; Lin, H. Triple-mode emission of carbon dots: Applications for advanced anti-counterfeiting. Angew. Chem. Int. Ed. 2016, 55, 7231-7235. [CrossRef] [PubMed]

33. Deng, Y.; Zhao, D.; Chen, X.; Wang, F.; Song, H.; Shen, D. Long lifetime pure organic phosphorescence based on water soluble carbon dots. Chem. Commun. 2013, 49, 5751. [CrossRef] [PubMed]

34. Li, W.; Wu, S.; Xu, X.; Zhuang, J.; Zhang, H.; Zhang, X.; Hu, C.; Lei, B.; Kaminski, C.F.; Liu, Y. Carbon dot-silica nanoparticle composites for ultralong lifetime phosphorescence imaging in tissue and cells at room temperature. Chem. Mater. 2019, 31, 9887-9894. [CrossRef]

35. Gou, H.; Liu, Y.; Zhang, G.; Liao, Q.; Huang, X.; Ning, F.; Ke, C.; Meng, Z.; Xi, K. Lifetime-tunable room-temperature phosphorescence of polyaniline carbon dots in adjustable polymer matrices. Nanoscale 2019, 11, 18311-18319. [CrossRef] 
36. Gao, Y.; Zhang, H.; Jiao, Y.; Lu, W.; Liu, Y.; Han, H.; Gong, X.; Shuang, S.; Dong, C. Strategy for activating room-temperature phosphorescence of carbon dots in aqueous environments. Chem. Mater. 2019, 31, 7979-7986. [CrossRef]

37. Jiang, K.; Gao, X.; Feng, X.; Wang, Y.; Li, Z.; Lin, H. Carbon dots with dual-emissive, robust, and aggregation-induced room-temperature phosphorescence characteristics. Angew. Chem. Int. Ed. 2020, 59, 1263-1269. [CrossRef]

38. Zhu, J.; Bai, X.; Chen, X.; Shao, H.; Zhai, Y.; Pan, G.; Zhang, H.; Ushakova, E.V.; Zhang, Y.; Song, H.; et al. Spectrally tunable solid state fluorescence and room-temperature phosphorescence of carbon dots synthesized via seeded growth method. Adv. Opt. Mater. 2019, 7, 1801599. [CrossRef]

39. Tao, S.; Lu, S.; Ge, J.; Zhu, S.; Redfern, S.A.T.; Song, Y.; Feng, T.; Xu, W.; Yang, B. Design of metal-free polymer carbon dots: A new class of room-temperature phosphorescent materials. Angew. Chem. Int. Ed. 2018, 57, 2393-2398. [CrossRef]

40. Long, P.; Feng, Y.; Cao, C.; Li, Y.; Han, J.; Li, S.; Peng, C.; Li, Z.; Feng, W. Self-protective room-temperature phosphorescence of fluorine and nitrogen codoped carbon dots. Adv. Funct. Mater. 2018, 28, 1800791. [CrossRef]

41. Jiang, K.; Wang, Y.; Gao, X.; Cai, C.; Lin, H. Facile, quick, and gram-scale synthesis of ultralong-lifetime room-temperature-phosphorescent carbon dots by microwave irradiation. Angew. Chem. Int. Ed. 2018, 57, 6216-6220. [CrossRef] [PubMed]

42. Jiang, K.; Wang, Y.; Cai, C.; Lin, H. Conversion of carbon dots from fluorescence to ultralong room-temperature phosphorescence by heating for security applications. Adv. Mater. 2018, 30, 1800783. [CrossRef] [PubMed]

43. Yuan, T.; Yuan, F.; Li, X.; Li, Y.; Fan, L.; Yang, S. Fluorescence-phosphorescence dual emissive carbon nitride quantum dots show 25\% white emission efficiency enabling single-component WLEDs. Chem. Sci. 2019, 10, 9801-9806. [CrossRef] [PubMed]

44. Li, H.; Ye, S.; Guo, J.; Kong, J.; Song, J.; Kang, Z.; Qu, J. The design of room-temperature-phosphorescent carbon dots and their applicationas a security ink. J. Mater. Chem. C 2019, 7, 10605-10612. [CrossRef]

45. Han, Y.; Li, M.; Lai, J.; Li, W.; Liu, Y.; Yin, L.; Yang, L.; Xue, X.; Vajtai, R.; Ajayan, P.M.; et al. Rational design of oxygen-enriched carbon dots with efficient room-temperature phosphorescent properties and high-tech security protection application. ACS Sustain. Chem. Eng. 2019, 7, 19918-19924. [CrossRef]

46. Wang, Z.; Liu, Y.; Zhen, S.; Li, X.; Zhang, W.; Sun, X.; Xu, B.; Wang, X.; Gao, Z.; Meng, X. Gram-scale synthesis of $41 \%$ efficient single-component white-light-emissive carbonized polymer dots with hybrid fluorescence/phosphorescence for white light-emitting diodes. Adv. Sci. 2020, 7, 1902688. [CrossRef]

47. Knoblauch, R.; Bui, B.; Raza, A.; Geddes, C.D. Heavy carbon nanodots: A new phosphorescentcarbon nanostructure. Phys.Chem.Chem.Phys. 2018, 20, 15518-15527. [CrossRef]

48. Feng, X.; Jiang, K.; Zeng, H.; Lin, H. A Facile approach to solid-state white emissive carbon dots and their application in uv-excitable and single-component-based white LEDs. Nanomaterials 2019, 9, 725. [CrossRef]

49. Gao, Y.; Han, H.; Lu, W.; Jiao, Y.; Liu, Y.; Gong, X.; Xian, M.; Shuang, S.; Dong, C. Matrix-free and highly efficient room-temperature phosphorescence of nitrogen-doped carbon dots. Langmuir 2018, 34, 12845-12852. [CrossRef]

50. Chen, Y.; Lian, H.; Wei, Y.; He, X.; Chen, Y.; Wang, B.; Zeng, Q.; Lin, J. Concentration-induced multi-colored emissions in carbon dots: Origination from triple fluorescent centers. Nanoscale 2018, 10, 6734-6743. [CrossRef]

51. Malyukin, Y.; Viagin, O.; Maksimchuk, P.; Dekaliuk, M.; Demchenko, A. Insight into the mechanism of the photoluminescence of carbon nanoparticles derived from cryogenic studies. Nanoscale 2018, 10, 9320-9328. [CrossRef]

52. Wang, Y.; Kalytchuk, S.; Zhang, Y.; Shi, H.; Kershaw, S.V.; Rogach, A.L. Thickness-dependent full-color emission tunability in a flexible carbon dot ionogel. J. Phys. Chem. Lett. 2014, 5, 1412-1420. [CrossRef]

53. Nie, H.; Li, M.; Li, Q.; Liang, S.; Tan, Y.; Sheng, L.; Shi, W.; Zhang, S.X.-A. Carbon dots with continuously tunable full-color emission and their application in ratiometric $\mathrm{pH}$ sensing. Chem. Mater. 2014, 26, 3104-3112. [CrossRef]

54. He, Z.; Zhao, W.; Lam, J.W.Y.; Peng, Q.; Ma, H.; Liang, G.; Shuai, Z.; Tang, B.Z. White light emission from a single organic molecule with dual phosphorescence at room temperature. Nat. Commun. 2017, 8, 416. [CrossRef] [PubMed] 
55. Hou, J.; Wang, L.; Zhang, P.; Xu, Y.; Ding, L. Facile synthesis of carbon dots in an immiscible system with excitation-independent rmission and thermally activated delayed fluorescence. Chem. Commun. 2015, 51, 17768-17771. [CrossRef] [PubMed]

56. Wang, Z.; Yuan, F.; Li, X.; Li, Y.; Zhong, H.; Fan, L.; Yang, S. 53\% efficient red emissive carbon quantum dots for high color rendering and stable warm white-light-emitting diodes. Adv. Mater. 2017, 29, 1702910. [CrossRef]

57. Chen, Y.; Zheng, M.; Xiao, Y.; Dong, H.; Zhang, H.; Zhuang, J.; Hu, H.; Lei, B.; Liu, Y. A self-quenching-resistant carbon-dot powder with tunable solid-state fluorescence and construction of dual-fluorescence morphologies for white light-emission. Adv. Mater. 2016, 28, 312-318. [CrossRef]

58. Zhu, S.; Zhang, J.; Wang, L.; Song, Y.; Zhang, G.; Wang, H.; Yang, B. A General route to make non-conjugated linear polymers luminescent. Chem. Commun. 2012, 48, 10889-10891. [CrossRef]

59. Pan, L.; Sun, S.; Zhang, A.; Jiang, K.; Zhang, L.; Dong, C.; Huang, Q.; Wu, A.; Lin, H. Truly fluorescent excitation-dependent carbon dots and their applications in multicolor cellular imaging and multidimensional sensing. Adv. Mater. 2015, 27, 7782-7787. [CrossRef]

60. Li, L.; Zhang, R.; Lu, C.; Sun, J.; Wang, L.; Qu, B.; Li, T.; Liu, Y.; Li, S. In situ synthesis of nir-light emitting carbon dots derived from spinach for bio-imaging applications. J. Mater. Chem. B 2017, 5, 7328-7334. [CrossRef]

61. Lu, S.; Sui, L.; Liu, J.; Zhu, S.; Chen, A.; Jin, M.; Yang, B. Near-infrared photoluminescent polymer-carbon nanodots with two-photon fluorescence. Adv. Mater. 2017, 29, 1603443. [CrossRef] [PubMed]

62. Du, Q.; Zheng, J.; Wang, J.; Yang, Y.; Liu, X. The synthesis of green fluorescent carbon dots for warm white LEDs. RSC Adv. 2018, 8, 19585-19595. [CrossRef]

63. Zhu, S.; Song, Y.; Zhao, X.; Shao, J.; Zhang, J.; Yang, B. The photoluminescence mechanism in carbon dots (graphene quantum dots, carbon nanodots, and polymer dots): current state and future perspective. Nano Res. 2015, 8, 355-381. [CrossRef]

64. Krysmann, M.J.; Kelarakis, A.; Dallas, P.; Giannelis, E.P. Formation mechanism of carbogenic nanoparticles with dual photoluminescence emission. J. Am. Chem. Soc. 2012, 134, 747-750. [CrossRef]

(C) 2020 by the authors. Licensee MDPI, Basel, Switzerland. This article is an open access article distributed under the terms and conditions of the Creative Commons Attribution (CC BY) license (http://creativecommons.org/licenses/by/4.0/). 